\title{
Sex hormones and mental health
}

\section{Louise Golightly \& Allan Young}

Hormones are widely considered to cause powerful psychological effects and because of this psychiatrists may be asked to advise general practitioners, consultants in other specialities or patients about the relationship between hormones (including sex hormones) and psychiatric disorder. Sex hormones may be relevant to psychiatry in three ways:

(a) Sex hormones may play a role in the pathophysiology of psychiatric disorders.

(b) These agents may be utilised for treatment of psychiatric disorder.

(c) Administration of sex hormones may cause psychiatric side-effects.

Of these three possibilities, psychiatrists are most frequently asked about adverse side-effects of sex hormones. This, therefore, will be the focus of this article. We will discuss these in relation to the common sex hormone preparations, which are: oral contraceptives; hormone replacement therapy; and androgens and anabolic-androgenic steroids.

\section{Oral contraceptives}

The predominant psychiatric side-effect in users of oral contraceptives is that of mood change; nonaffective psychiatric side-effects of sex hormones are rare. Reports of the effects of oral contraceptives on mood began to emerge soon after they were introduced in the 1960s and since then various effects have been described. Some studies have implicated oral contraceptives in causing depression, with suggested mechanisms including induction of abnormal biochemical changes and vitamin deficiencies. Some researchers have considered oestrogens to be responsible for these mood changes, whereas others have postulated that progestogens are causative. Other studies, however, have shown no such adverse effects of oral contraceptives, while still further studies have reported elevated mood in users of oral contraceptives. In considering the apparent incidence of mood changes in oral contraceptive users it is necessary to determine whether the observed mood changes reflect preexisting psychiatric morbidity or the social and psychological effects of using a contraceptive. Methodological problems in some studies may be responsible for conflicting results, for example, prospective versus retrospective studies, small sample size, the relative doses of oestrogen and progestogen in the preparation used, and the rating scales used to assess mood changes. Finally, several authors have suggested that the 'depression' complained of by some users of oral contraceptives is different to that recognised by psychiatrists as true affective disorder, in that it is atypical in presentation or does not fulfil diagnostic criteria.

Before examining the effects on mood of oral contraceptives it is necessary briefly to review the different types of oral contraceptives and their mechanism of action. The rise of oral contraceptives was made possible by the development of synthetic oestrogens and progestogens which have higher potency than natural steroids.

There are two main types of oral contraceptives: combined and progestogen-only. Combined pills contain both oestrogen and progestogen and are taken for 21 days. During the next seven pill-free days steroid levels fall and a withdrawal bleed occurs. Progestogen-only pills contain the same dose throughout the cycle and are taken continuously. The most commonly used oestrogen is ethinyloestradiol and the most commonly used progestogens are norethisterone and levonorgestrel.

Allan Young is a Senior Lecturer in Neurosciences and Psychiatry and an Honorary Consultant in Psychiatry at the Department of Psychiatry at the University of Newcastle (The Royal Victoria Infirmary, Queen Victoria Road, Newcastle upon Tyne NE1 4LP). His research interests are neuroscience, affective disorders and psychotic disorders. Louise Golightly is a Specialist Registrar and Clinical Research Associate in the Department of Psychiatry, University of Newcastle. She has clinical and research interests in schizophrenia and psychoneuroendocrinology. 
Combined preparations of oral contraceptives work primarily by suppression of ovulation, with the secondary effects on cervical mucus reducing sperm penetration and elsewhere in the reproductive tract further reducing fertility. Progestogen-only pills rely on their endometrial effects and their effects on cervical mucus.

\section{Mood changes}

The feeling of improved well-being and increased activity experienced by some women using oral contraceptives has been attributed to oestrogen, which inhibits monoamine oxidase activity leading to increased availability of catecholamines and indoleamines. This effect has also been attributed to pro-gestational agents, possibly through their sedative action in the presence of stress: Worsley (1980) correlated higher progestogen doses with improved mood scores compared with control subjects. Improved mood has also been attributed to a specific psychological factor associated with oral contraceptives, that is, relief from the fear of pregnancy.

The depressant mechanism of action of oral contraceptives is thought to be a pharmacological effect, either of the oestrogen component (by increasing metabolism of tryptophan) or of the progestogen part (which elevates monoamine oxidase at the synaptic junction). However, Warnes \& Fitzpatrick (1979) showed that changes in urinary serotonin and catecholamine metabolites associated with oral contraceptive use did not correlate with depressive symptomatology. It seems, therefore, that depressive mood may be associated, at least in part, with non-pharmacological aspects of using contraceptives.

In an early study of the effect of oral contraceptives on depressive mood changes (Grant \& Pryce-Davies, 1968) significant variations in the incidence of depression with various oral contraceptives were found. The rate of depression was higher with strongly progestogenic compounds, especially when these had low doses of oestrogens, while the lowest incidence of depression was found with strongly oestrogenic sequential regimens. The rate of depression was also found to be related to endometrial monoamine oxidase activity. The incidence of depression ranged from $28 \%$ of women on the strongly progestogenic preparations to $7 \%$ of women on strongly oestrogenic regimens. However, Kutner \& Brown (1972) found opposite effects with pills containing high doses of progestogens causing less severe depression. Cullberg (1973) examined the effects on mood changes with different progestogen/ oestrogen combinations, and found that all oral contraceptives produced significantly more adverse psychological effects than placebo, but that the effects were independent of the dose of progestogen in the preparation. It should be noted that Grant \& Pryce-Davies (1968) did not use standardised rating scales to assess depression, and many of the early studies do not specify operational criteria for the diagnosis of depression.

Bancroft et al (1987) considered a further factor that may determine the effects on mood of oral contraceptives, that is, the fact that women may experience different effects on mood at different stages of their use of oral contraceptives. They randomly allocated women who were due to start taking an oral contraceptives to either a combined or a triphasic pill. The degree of cyclical mood change was assessed prior to starting the pill and they were then divided into two groups: those with and those without premenstrual mood change. For women with no history of premenstrual mood change the type of pill made no difference to mood. However, for those with premenstrual mood change, those taking the triphasic pill experienced significantly more lowering of mood than those taking the combined preparation. This finding was consistent with that of Cullberg (1973) described above. There is a need, therefore, to distinguish between the initial effects of starting the pill and later effects on women taking oral contraceptives in the long term. Between the early and late phases some women will stop the pill, possibly because of side-effects. Studies of long-term users will, therefore, have already selected out some women experiencing side-effects. Also, during the initial phase, the brain must readjust to a new cyclical hormone regime, which may have effects on mood.

Several studies have found that previous history of depression was positively correlated with depression with oral contraceptives; unfortunately, these did not use control subjects. Kutner \& Brown (1972) studied a history of depression during or after pregnancy as a risk factor for depression with oral contraceptives in current and past users of oral contraceptives. They found no evidence of oral contraceptives aggravating a depressive history.

Chang et al (1982) aimed to clarify the conflicting results of previous studies and eliminate some of the problems of rating depression in these women. They used the method of factor analysis to produce a questionnaire that reliably assessed the type of emotional symptoms experienced by women using oral contraceptives. This demonstrated the multi-dimensional nature of reported emotional side-effects from the pill, and these authors concluded that it seems doubtful that depression reported by women taking the pill is the same as depression as defined operationally in psychiatry, or that it can be meaningfully measured using standard rating scales for depression. 
Overall, there are conflicting reports of the nature of mood changes, if any, caused by the use of oral contraceptive pills. In a recent review, Patten \& Love (1994) comment that there is no evidence that a past or family history of depression is a contraindication to the use of oral contraceptives. They further comment that given the paucity of evidence that oral contraceptives can cause depression, it is not surprising that there are few published guidelines on the management of oral contraceptive-induced depression (see Box 1).

\section{Hormone replacement therapy}

Hormone replacement therapy (HRT) is designed to increase oestrogen levels and so prevent or treat clinical features associated with the menopause. It can be given whether the menopause occurs naturally or following chemotherapy or surgical removal of the ovaries. Systemic oestrogens may cause endometrial hyperplasia and hence carcinoma, and are therefore given with a progestogen, which protects against these changes.

The primary components of most HRT preparations are one or more natural oestrogens, for example, oestradiol, oestrogen and oestriol. Synthetic progestogens are used, for example, dydrogesterone, medroxyprogesterone acetate, levonorgestrel, norgestrel and norethisterone.

There appears to be little in the literature relating to adverse psychiatric effects of HRT. In fact, an article about HRT in the Drug and Therapeutics Bulletin in November 1996 (Anonymous, 1996) made no specific mention of psychiatric effects and only alluded to this in stating that symptoms similar to those of the premenstrual syndrome (progestogen-

Box 1. Mood changes with oral contraceptives

It is not clear to what extent (if at all) oral contraceptives cause mood changes

Mood changes, if they occur, may be related to the ratio of oestrogen to progestogen dose

Psychological as well as biological effects should be considered in women apparently presenting with oral contraceptive-induced depression

Past or family history of depression is not a contraindication to the use of oral contraceptives related effects) may occur. Furthermore, reference to the ABPI Data Sheet Compendium (Walker, 1998) lists only depression as a rare adverse effect, if at all, in most preparations. On the contrary, there is much to suggest that HRT, and specifically oestrogens, improve psychological well-being in post-menopausal women. This may be secondary to its beneficial effects on other symptoms such as vasomotor symptoms. However, specific effects have been suggested. For instance, Lippert et al (1996) showed that oestradiol produces a significant increase in urinary 5hydroxy-3-indole acetic acid excretion and comments that enhancement of serotonin turnover might contribute to improvement in mood and well-being.

\section{Effects on psychological well-being}

Figures of the incidence of depressed mood in menopausal women vary. However, some studies have shown that up to one-third of menopausal women report elevated levels of depressed mood. Therefore, improvement in psychological functioning, especially depressed mood, may be of great significance. There is a theoretical rationale for a relationship between HRT and depressed mood. For example, Sherwin \& Gelfand (1985) proposed that oestrogen replacement alleviates depressed mood among menopausal women by increasing serotonin levels. There has, however, been inconsistency in the outcomes reported in studies of the effects of HRT on mood, due to methodological problems such as small sample size, varying levels of depression, differing and non-standardised depression ratings used and the type, dose and length of treatment of HRT. Zweifel \& O'Brien (1997)_performed a metaanalysis of the effect of HRT on depressed mood, which suggested substantial and significant effect sizes associated with HRT producing a reduction in depressed mood. It also indicated that the addition of progesterone to the oestrogen treatment reduced the beneficial effect of HRT on depressed mood and that androgen alone and in combination with oestrogen was associated with greater reductions in depressed mood. Most subjects in the study were not significantly depressed prior to treatment, however, limiting the probability of an improvement with HRT; therefore, the effect sizes estimated in this meta-analysis may underestimate the true effectiveness of HRT for patients with significant levels of depression.

Zweifel's study, however, left some unanswered questions such as the optimal combination of hormones, the relationship of effectiveness to demographic variables, dose-response relationships and the variation of outcomes with menopausal status, type of menopause and severity of pre- 
treatment symptoms. Some of these issues have been addressed by other studies. Klaiber et al (1996) carried out a double-blind placebo-controlled crossover study of changes in mood during HRT in non-depressed menopausal women. They comment that oestrogen alone enhances mood states, but that combination of oestrogen plus progestogen induces negative mood changes and that this effect may be due to opposite effects of oestrogen and progestogen on serotonergic and adrenergic functions. However, these opposite effects are not seen in all menopausal women and so Klaiber set out to identify which factors determine who will be adversely affected. Their findings were that administration of oestrogen alone tends to improve mood, while addition of progestogen diminishes the positive effects of oestrogen alone. Also, not all subjects respond in the same manner to HRT. Trend analyses were then performed to identify the characteristics associated with different patterns of response to HRT. Adverse mood effects to progestogen were found to occur in women with: (a) a long duration of menopause; (b) low pre-treatment serum oestradiol and testosterone levels; (c) high pre-treatment follicle-stimulating hormone levels; (d) low pre-treatment monoamine oxidase activity; and (e) pre-treatment mood abnormalities, with individuals with adverse pretreatment moods experiencing more positive mood responses to oestrogen alone that are not completely countered by negative responses to the addition of a progestogen. They conclude that adverse mood responses to the addition of progestogen occur in menopausal women with low pre-treatment gonadal hormone levels secondary to a menopause of long duration, and that impaired central nervous system (CNS) adrenergic and serotonergic functioning also may be a factor predisposing to a negative mood response to progestogen.

Several other studies have confirmed the finding of improved mood in menopausal women taking HRT, particularly three studies by Sherwin (1988, 1991, 1996). She hypothesises that oestrogen activity in the limbic system together with its effects on neurotransmitter activity in the CNS improves psychological functioning (Sherwin, 1996), a suggestion which is also supported by some preclinical data (Young et al, 1993). In a study of healthy surgically menopausal women (Sherwin, 1988) receiving either intramuscular oestrogen-androgen combined preparations or oestrogen alone long-term, the women receiving treatment had more positive moods than the untreated control subjects, coincident with higher circulating oestradiol levels. This confirmed that mood varies with circulating oestradiol levels in generally healthy non-depressed women. A further study by Sherwin (1991) investigated the effects of various doses of oestrogen and progestogen in healthy naturally menopausal women. This study found that the effects of progestogen on the CNS are reflected by an increase in psychological symptomatology and are attenuated by higher oestrogen/ progestogen ratio. Again this suggests that oestrogen in HRT preparations tends to improve mood in menopausal women. Ditkoff et al (1991) also demonstrated, in a randomised double-blind study of the effects of oestrogens on psychological function in asymptomatic post-menopausal women, that oestrogen might improve the overall quality of life, including mood, in post-menopausal women.

However, it should be noted that not all studies have been so enthusiastic in their recommendations for the use of HRT. Hunter (1990) in a review article stated that although oestrogen may have a "mental tonic" effect when prescribed in high doses, HRT does not appear to have a significant effect upon mood over and above placebo effects. Furthermore, studies of menopausal women who meet criteria for depressive disorder have not demonstrated significant improvement in response to oestrogen replacement therapy at typical doses and may require treatment with antidepressant medication.

The overall impression of the effects of HRT on psychiatric symptoms would appear to be an improvement in mood and general psychological wellbeing. The cautions to this would be that this appears to depend on the relative oestrogen/progestogen dose of the preparation and on the pre-treatment depression levels of the patient (see Box 2).

\section{Androgens and anabolic- androgenic steroids}

Androgens are steroid hormones with two different effects: androgenic effects, that is differentiation, growth and development of the male reproductive

Box 2. Mood changes with HRT

The general impression is that HRT produces an improvement in mood and general psychological well-being in menopausal women

The effects depend on the oestrogen/progestogen ratio of the preparation used

Pre-treatment mood levels may influence the likelihood of positive effects of HRT

No significant improvement has been shown in women who are clinically depressed 
tract; and anabolic effects, that is stimulation of linear body growth and somatic growth. During early development, androgens masculinise internal and external genitalia and the brain. After puberty, masculinising effects consist of maturation of external genitalia and accessory sex organs, stimulation of beard and axillary and pubic hair, temporal hair recession, balding, enlargement of the larynx and thickening of the vocal cords, facilitation of libido and sexual potency. The primary use of anabolic-androgenic steroids (AASs) is in replacement therapy for male hypogonadism; other medical uses include growth promotion in various forms of stunted growth, osteoporosis, mammary carcinoma, anaemias and hereditary angioneurotic oedema. Aside of their clinical use, these drugs are widely misused among athletes to promote body size and muscle strength and it is estimated that there are over a million users in the USA. Major mood disturbances associated with AAS use may represent an important public health problem for athletes using steroids and sometimes for the victims of their irritability and aggression.

\section{AASs and psychiatric illness}

It was Charles Brown-Sequard's experiment in 1889 that heralded the possibility that testosterone and its relatives, AASs, might have mental effects. He described the salutary effects on his health of selfinjections of extracts of crushed animal testicles, that is, energy, muscular strength, stamina and mental agility. Pure preparations of testosterone became available in the late 1930s and investigators began to explore the hormone's potential therapeutic effects in various medical conditions, especially psychotic and involutional disorders. Several studies (reviewed by Pope \& Katz, 1992) reported striking improvements in patients with various affective disorders when treated with testosterone and other androgens, although other studies reported less success. Overall, there appear to be approximately four times as many positive as negative reports describing the efficacy of testosterone in depression, including a recent double-blind placebo-controlled trial (Morales et al, 1994). The review by Pope \& Katz also describes reports claiming success with testosterone or related androgens in schizophrenia and anorexia nervosa. However, these studies were difficult to interpret owing to their uncontrolled or anecdotal nature. The development of effective drug treatments for affective and psychotic disorders in the 1950s and 1960s may have influenced the relative lack of newer, better-controlled studies with steroids in psychiatric disorders.

Clinical studies using physiological or only moderately supra-physiological doses of steroids have rarely noted prominent psychiatric effects. In a large and thorough review, Bahrke et al (1990) comment that studies in individuals with androgen deficiencies receiving androgen therapy give mixed results, with only some demonstrating significant positive psychological changes with AASs. However, no adverse or undesirable psychological effects were observed. There are, however, reports of patients developing psychotic disorders in response to treatment with physiological doses. For example, Annitto \& Layman (1980) reported the case of a 17year-old male with no previous psychiatric history who developed a schizophreniform illness on two separate occasions after commencing regular misuse of methandienone obtained on the black market. The illness resolved spontaneously after discontinuation of the drug on each occasion. Pope \& Katz (1988) reported the case of a 40-year-old man prescribed methyltestosterone for idiopathic impotence who developed severe depression with vegetative and psychotic symptoms within two weeks of starting the drug. These subsided after discontinuation of the drug.

\section{AAS use in athletes}

The vast majority of psychiatric effects of AAS use are in athletes using very high doses. Athletes often use doses up to 100 times the recommended therapeutic dose and use several drugs at the same time ('stacking') including using combinations of oral and injectable preparations. In general, steroid exposure appears to produce mania-like features including irritability, aggressiveness, euphoria, grandiose beliefs, hyperactivity and reckless behaviour. Psychotic symptoms have been reported, as have episodes of serious violent behaviour including cases of attempted and successful murder by people using AASs with no previous psychiatric or criminal history.

There are many methodological problems with studies of the psychiatric effects of AAS use in athletes and these will be described here before reviewing some of the studies. First, studies are usually retrospective and non-blind and subjects' retrospective reports of the effects of illicitly obtained drugs are of uncertain authenticity. Often, there is no urine testing to confirm which steroids or other drugs are being used, resulting in unsystematic studies regarding drugs and doses used. Selfselection of subjects in response to advertisements may produce subjects who are not representative of the overall population of steroid users. It is often unclear whether reported psychiatric syndromes are due to steroid use or to other psychoactive drugs, pre-existing psychiatric disorders, expectancy effects 
or social influences from the gymnasium subculture. A variety of psychological inventories have been used making comparability of findings between studies difficult. Furthermore, in some cases, nonstandardised and unpublished inventories have been used. In addition, some inventories measure trait and not state characteristics, so that testosterone levels may be correlated with a temporary state rather than an enduring personality characteristic. Attempts to use controls are also unsatisfactory: control groups of non-steroid-using athletes leave the question of whether the differences observed are steroid effects or antecedent psychological differences between users and non-users; using subjects as their own controls and comparing symptoms on and off steroids is retrospective and open to recall bias on the part of the subject. Ethical considerations prevent mimicking illicit drug use by administering massive doses of several steroids to volunteers in a double-blind fashion, and so only naturalistic study designs can be used. Nevertheless, the fairly consistent results tend to conclude that steroid use and withdrawal do produce prominent affective symptoms in at least some athletes and these are sometimes sufficient to cause morbidity and even mortality.

One study which was double-blind, placebocontrolled and prospective was performed in 20 normal male volunteers by Su et al (1993). This was a crossover trial of methyltestosterone, assessing mood and behaviour, and found significantly increased symptom scores during high-dose methyltestosterone compared with baseline in positive mood, negative mood and cognitive impairment. One subject became manic and one hypomanic. Family psychiatric history and previous drug misuse did not predict symptom changes.

In 1988, to assess the frequency of affective and psychotic symptoms in athletes taking AASs, Pope \& Katz performed structured interviews of 41 bodybuilders and football players who had used AASs. According to DSM-III-R (American Psychiatric association, 1987), $22 \%$ displayed a full affective syndrome with $12 \%$ displaying psychotic symptoms. Twelve per cent met DSM-III-R criteria for manic episodes with a further $19 \%$ narrowly failing to meet these criteria. Twelve per cent of subjects developed major depression when withdrawing from steroids. Subjects' past psychiatric histories were unremarkable. The authors comment that these findings should be seen as descriptive rather than quantitative, since it is unclear whether their observations were representative of the experiences of steroid users as a whole.

An investigation by Perry et al (1990) characterises the symptom patterns and mental status changes precipitated by anabolic steroid use. Twenty male weight-lifters currently using AASs were compared with 20 male weight-lifters who had never used steroids. Steroid users had significantly more somatic, depressive, anxiety, hostility and paranoid complaints when using steroids than when not using them. Compared with the weight-lifter control group, steroid users had a significantly greater number of complaints of depression, anxiety and hostility during cycles of steroid use. However, no differences in the frequency of major mental disorders were found between the two groups. The authors concluded that organic affective changes associated with AAS use usually present as a subsyndromal depressive disorder of insufficient severity to be classified as a psychiatric disorder.

Moss et al (1992) assessed the relationship between anabolic steroids and specific personality dimensions in 50 male body-builders who were current or past users compared with a sample of 25 'natural' male body-builders who never used AASs. No personality differences were found. The relationship between current AAS use and the presence of variations in mood state, hostility and psychiatric symptomatology was evaluated. Current AAS users scored higher than non-users only on psychometric scales measuring hostility, aggression and somatisation, therefore, this study could not confirm reports that AAS use was associated with significant psychopathology, other than aggression and somatisation. This study, however, did not use urine toxicology and did not record the doses of AASs used.

A further study by Pope \& Katz (1994) compared 88 athletes using steroids with 68 non-users using the Structured Clinical Interview for DSMIII-R (Spitzer et al, 1990). Twenty-three per cent of steroid users reported major mood syndromes, that is mania, hypomania or depression. These included cases with psychotic symptoms and aggressive or violent behaviour often accompanied steroidassociated manic or hypomanic episodes. Steroid users displayed mood disorders during steroid exposure significantly more frequently than in the absence of steroid exposure and significantly more frequently than non-users. A highly significant relationship was found between total weekly dose of steroids used and the prevalence of mood disorders.

\section{Psychological dependence and withdrawal effects}

The continued use of AASs is motivated by the creation of a new body-image, improved self-esteem, heightened libido and general euphoria. Discon- 
tinuation of AAS use results in an often dramatic reduction in size, weight and strength. Withdrawal depression may also promote dependence and increase the possibility of suicide. Yesalis et al (1989) described approximately $25 \%$ of adolescent AAS users reporting behaviours, perceptions and opinions consistent with psychological dependence. Withdrawal effects include mood swings, violent behaviour, rage and depression. Brower et al (1989) report a case where tolerance, withdrawal symptoms and the use of AASs to alleviate withdrawal symptoms had occurred. Steroid use continued despite severe mood disturbance, marital conflict and changes in the patient's usual values.

It seems, therefore, that therapeutic doses of androgens in clinical use rarely cause significant psychiatric effects. However, there is a wealth of literature describing affective disorders and aggressive behaviour in athletes using extremely high doses of multiple AASs to promote size, weight and strength, and there is mounting evidence of dependence on these drugs. Nevertheless, the fact that misuse of these drugs is so common means that either the risk of developing psychiatric effects is low, or that sufferers do not seek professional help, or that this important aetiological factor is being missed when AAS-induced mental illness presents (Williamson \& Young, 1992). As described above, the study of the effects of these drugs is filled with methodological and ethical problems but could possibly clarify the complex interactions between hormones, behaviour and the development of mental illness (see Box 3).

\section{Premenstrual syndrome}

Although premenstrual syndrome (PMS) does not fall within the focus of this article as defined in the introduction, any article discussing the psychiatric effects of sex hormones would be incomplete without reference to this condition. Premenstrual syndrome consists of mood-related and somatic symptoms occurring during the luteal phase of the menstrual cycle and disappearing at or soon after the onset of menstruation in the absence of ovarian dysfunction. Because of the prominence of mood symptoms in this syndrome, psychiatrists are often asked to contribute to its treatment. Effective treatments include selective serotonin reuptake inhibitors, alprazolam and gonadotropin-releasing hormone agonists such as leuprorelin acetate (Mortola, 1998). It has been estimated using strict diagnostic criteria that the prevalence of the

\section{Box 3. Psychiatric effects of AASs}

Recent literature suggests a high incidence of psychiatric morbidity in AAS users, with some users exhibiting addictive behaviour

Withdrawal depression may promote dependence and increase the possibility of suicide

There is accumulating evidence that steroidinduced psychiatric effects may represent a public health problem as anabolic steroids can increase violent or criminal behaviour

disorder is $2.5 \%$. The pathophysiological role of the luteal phase is unclear but it seems that PMS is triggered by hormone-related events occurring before the mid-luteal phase of the menstrual cycle. A recent study by Schmidt et al (1998) attempted to determine the role of oestrogen and progesterone in the syndrome. They studied the effect of ovarian suppression with the agonist analogue of the gonadotrophin-releasing hormone, leuprorelin, and then of treatment with alternately progesterone and oestrogen during continued leuprorelin administration. They found that women with PMS given leuprorelin had significant reduction in PMS symptoms compared to baseline and to placebo. Women with PMS given leuprorelin plus oestradiol or progesterone had significant recurrence of their symptoms. There were no changes in normal control women receiving the same treatment or in women given placebo during continued leuprorelin treatment. They concluded that in women with PMS, the occurrence of symptoms represents an abnormal response to normal hormonal changes. Further studies are necessary to clarify the pathophysiology of PMS. However, should the role of oestrogen in the symptoms of PMS be confirmed, this would allow the development of new treatments based on drugs or hormones with anti-oestrogenic properties.

\section{Conclusions}

The most common psychiatric effects of sex hormone preparations are on mood, although it must be emphasised that in many cases there will be no adverse effect of these drugs when used at therapeutic dosage levels. Furthermore, when there is an effect, this may be a positive effect on 
psychological well-being. The nature of the effect on mood may be related to the type of preparation, the ratio of oestrogen to progestogen (in the case of HRT and oral contraceptive preparations), the pre-existing mood state and personality traits of the individual. In the case of athletes who misuse AASs, the adverse effects on mood and behaviour may amount to a serious problem for the user and those who suffer the consequences of their behaviour. When considering whether to prescribe hormonal preparations there will rarely be a psychiatric contraindication and the physical indication for the drug should take precedence. However, treatment may need to be subsequently altered if a significant psychiatric side-effect develops.

\section{References}

American Psychiatric Association (1987) Diagnostic and Statistical Manual of Mental Disorders (3rd edn, revised) (DSM-III-R). Washington, DC: APA.

Anonymous (1996) Hormone replacement therapy. Drug and Therapeutics Bulletin, 34, 81-84.

Annitto, W. J. \& Layman, W. A (1980) Anabolic steroids and acute schizophrenic episode. Journal of Clinical Psychiatry, 41, 143-144.

Bahrke, M. S., Yesalis III, C. E. \& Wright, J. E. (1990) Psychological effects of endogenous testosterone levels and anabolic-androgenic steroids among males. Sports Medicine, 10, 303-337.

Bancroft, J., Sanders, D., Warner, P., et al (1987) The effects of oral contraceptives on mood and sexuality: a comparison of triphasic and combined preparations. Journal of Psychosomatic Obstetrics and Gynaecology, 7, 1-8.

Brower, K. J., Blow, F. C., Beresford, T. P., et al (1989) Anabolicandrogenic steroid dependence. Journal of Clinical Psychiatry, 50, 31-33.

Brown-Sequard, C. E. (1889) The effects produced on man by subcutaneous injection of a liquid obtained from the testicles of animals. Lancet, ii,105-107.

Chang, A. M. Z., Chick, P. \& Milburn, S. (1982) Mood changes as reported by women taking the oral contraceptive pill. Australian and New Zealand Journal of Obstetrics and Gynaecology, 22, 78-83.

Cullberg, J. (1973) Mood changes and menstrual symptoms with different gestagen/oestrogen combinations. Acta Psychiatrica Scandinavica, 236 (suppl.), 1-86.

Ditkoff, E. C., Crary, W. G., Cristo, M., et al (1991) Estrogen improves psychological function in asymptomatic postmenopausal women. Obstetrics and Gynecology, 78, 991-995.

Grant, E. C. \& Pryce-Davies, J. (1968) Effect of oral contraceptives on depressive mood changes and on endometrial monoamine oxidase and phosphatases. British Medical Journal, 3, 777-780.

Hunter, M. S. (1990) Emotional well-being, sexual behaviour and HRT. Maturitas, 12, 299-314.

Klaiber, E. L., Broverman, D. M., Vogel, W., et al (1996) Individual differences in changes in mood and platelet monoamine oxidase activity during hormonal replacement therapy in menopausal women. Psychoneuroendocrinology, 21, 575-592.

Kutner, S. J. \& Brown, W. L. (1972) History of depression as a risk factor for depression with oral contraceptives and discontinuance. Journal of Nervous and Mental Disease, 155, 163-169.
Lippert, T. H., Filshie, M., Muck, A. O., et al (1996) Serotonin metabolite excretion after post-menopausal estradiol therapy. Maturitas, 24, 37-41.

Morales, A. J., Nolan, J. J., Nelson, J. C., et al (1994) Effects of replacement dose of dehydroepiandrosterone in men and women of advancing age. Journal of Clinical Endocrinology and Metabolism, 78, 1360-1367.

Mortola, J. F. (1998) Premenstrual syndrome - pathophysiologic considerations. New England Journal of Medicine, 338, 256-257.

Moss, H. B., Panzak, G. L. \& Tarter, R. E. (1992) Personality, mood and psychiatric symptoms among anabolic steroid users. American Journal on Addictions, 1, 315-324.

Patten, S. B. \& Love, E. J. (1994) Drug-induced depression. Incidence, avoidance and management. Drug Safety, 10, 203-219.

Perry, P. J., Yates, W. R. \& Andersen, K. H. (1990) Psychiatric symptoms associated with anabolic steroids: a controlled retrospective study. Annals of Clinical Psychiatry, 2, 11-17.

Pope, H. G. \& Katz, D. L. (1988) Affective and psychotic symptoms associated with anabolic steroid use. American Journal of Psychiatry, 145, 487-490.

- \& - (1992) Psychiatric effects of anabolic steroids. Psychiatric Annals, 22, 24-29.

- \& - (1994) Psychiatric and medical effects of anabolicandrogenic steroid use. Archives of General Psychiatry, 51, 375-382.

Schmidt, P. J., Nieman, L. K., Danaceau, M. A., et al (1998) Differential behavioural effects of gonadal steroids in women with and in those without premenstrual syndrome. New England Journal of Medicine, 388, 209-217.

Sherwin, B. B. (1988) Affective changes with oestrogen and androgen replacement therapy in surgically menopausal women. Journal of Affective Disorders, 14, 177-187.

- (1991) The impact of different doses of oestrogen and progestin on mood and sexual behaviour in postmenopausal women. Journal of Clinical Endocrinology and Metabolism, 72, 336-343.

- (1996) Hormones, mood and cognitive functioning in postmenopausal women. Obstetrics and Gynecology, 87, 20S-26S.

- \& Gelfand, M. M. (1985) Sex steroids and affect in the surgical menopause: a double-blind, crossover study. Psychoneuroendocrinology, 10, 325-335.

Spitzer, R. L., Williams, J. B. W., Gibbon, M., et al (1990) User's Guide for the Structured Clinical Interview for DSM-III-R: SCID. Washington, DC: American Psychiatric Press.

Su, T., Pagliaro, M., Schmidt, P. J., et al (1993) Neuropsychiatric effects of anabolic steroids in male normal volunteers. Journal of the American Medical Association, 269, 2760-2764

Walker, G. (1998) Association of the British Pharmaceutical Industry Data Sheet Compendium. London: Datapharm Publications.

Warnes,H. \& Fitzpatrick, C. (1979) Oral contraceptives and depression. Psychosomatics, 20, 187-194.

Williamson, D. J. \& Young, A. H. (1992) Psychiatric effects of anabolic-androgenic steroid abuse in men: a brief review of the literature. Journal of Psychopharmacology, 6, 20-26.

Worsley, A. (1980) A prospective study of the effects of the progestogen content of oral contraceptives on measures of affect, automatization and perceptual restructuring ability. Psychopharmacologia, 67, 289-296.

Yesalis, C. E., Streit, A. L., Vicary, J. R., et al (1989) Anabolic steroid use: indications of habituation among adolescents. Journal of Drug Education, 19, 103-116.

Young, A. H., Dow, R. C., Goodwin, G., M., et al (1993) The effects of adrenalectomy and ovariectomy on the behavioural and hypothermic responses of rats to 8-Hydroxy2(Di-n-propylamino)tetralin. Neuropharmacology, 32, 653657.

Zweifel, J. E. \& O'Brien, W. H. (1997) A meta-analysis of the effect of hormone replacement therapy upon depressed mood. Psychoneuroendocrinology, 22, 189-212. 


\section{Multiple choice questions}

1. In considering the psychiatric effects of oral contraceptives:

a there is clear evidence that oral contraceptives cause depression

b observed mood changes may be due to psychological factors

c higher oestrogen/progestogen dose ratio is more likely to cause depression

d past history of depression is a contraindication to the prescription of oral contraceptives

e 'depression' caused by oral contraceptives may be qualitatively different from that recognised by psychiatrists.

\section{With respect to HRT:}

a there is no evidence for specific effects of HRT producing improved psychological well-being

b improved psychological well-being in women using HRT may be secondary to its effects on physical symptoms

c higher oestrogen/progestogen ratio preparations are more beneficial in improving psychological well-being in menopausal women

d women with low pre-treatment serum oestradiol and testosterone levels are more likely to experience adverse effects to progestogen

e pre-treatment depression levels of the patient have no influence on the effects of HRT on mood.

3. With regard to androgens:

a testosterone is recognised to be an effective adjuvant therapy for depression.

b therapeutic doses of androgens commonly cause adverse psychological effects

c athletes often use up to 100 times therapeutic doses of androgens

$\mathrm{d}$ addiction to anabolic-androgenic steroids (AASs) does not occur

e there is a risk of suicide in athletes discontinuing AAS use.
4. Which of the following is/are true?

a AAS use has been used as a legal defence against violent behaviour

b subjects with no past history of criminal behaviour have been known to commit murder when using AASs

c the first study to observe the mental effects of testosterone in man involved the injection of crushed animal testicles

$d$ the usual symptom pattern observed in users of AASs is one suggestive of mania

e commonly, AAS users experiencing adverse psychological effects do not seek professional help.

5. Which of the following is/are true?

a psychiatric side-effects of oral contraceptives, other than those relating to mood, are rare

b abnormal biochemical changes have been postulated as a cause for psychiatric sideeffects of oral contraceptives

c interpretation of studies of sex hormones may be complicated by methodological problems

d hormone replacement therapy should not be given to women experiencing menopausal symptoms as a result of hysterectomy

e depressed mood is rare in menopausal women. 